\title{
Transient heat generation in a quantum dot under a step-like pulse bias
}

\author{
Wei Pei, ${ }^{1}$ X. C. Xie, ${ }^{2,3}$ and Qing-feng Sun ${ }^{1, *}$ \\ ${ }^{1}$ Institute of Physics, Chinese Academy of Sciences, Beijing 100190, China \\ ${ }^{2}$ International Center for Quantum Materials, Peking University, Beijing 100871, China \\ ${ }^{3}$ Department of Physics, Oklahoma State University, Stillwater, Oklahoma 74078
}

\begin{abstract}
We study the transient heat generation in a quantum dot system driven by a step-like or a squareshaped pulse bias. We find that a periodically oscillating heat generation arises after adding the sudden bias. One particularly surprising result is that there exists a heat absorption from the zero-temperature phonon subsystem. Thus the phonon population in non-equilibrium can be less than that of the equilibrium electron-phonon system. In addition, we also ascertain the optimal conditions for the operation of a quantum dot with the minimum heat generation.
\end{abstract}

PACS numbers: $65.80 .-\mathrm{g}, 44.90 .+\mathrm{c}, 73.23 .-\mathrm{b}$

\section{INTRODUCTION}

With the technologic development in the microintegration of electronic devices, the heat generation problem has sharply emerged. This increasing thermal generation greatly influences the performance and stability of nanoscale electronic devices, and it becomes a main bottleneck for the future advancement of electronic industry. Furthermore, some familiar concepts of heat generation that are applicable in macroscopic systems may not be valid in nanoscale systems. So there is an imperative need to comprehend fully the heating effect and to try to mitigate it.

Owing to difficulties in probing the heating process in nanostructures, not much progress had been made until several state-of-the-art experimental scenarios were conceived in recent years $\underline{\underline{1}} \underline{\underline{-8}}$ There are both indirect $\underline{\underline{1}} \underline{\underline{6}}$ and direct $\frac{7.8}{}$ methods, which enable the evaluation of the effective local temperatures of nanoscale junctions. It has been experimentally demonstrated that the local heating may induce a substantial temperature increase in single molecular junctions ${ }^{3-\underline{6}}$ because of the inefficient heat dissipation. From a microscopic point of view, the main factor of heat generation is the electron-phonon (e-p) interaction, which transfers the ordered energy of electric current to the disordered vibrational energy of atoms. In some recent measurements of differential conductance, phonon-assisted tunnelling peaks have been observed in various nano-devices (e.g. $\mathrm{C}_{60}$, carbon nanotube, etc.) $\stackrel{9-15}{=}$ these clearly show the existence of e$\mathrm{p}$ interaction. As for the aspect of theory, on the one hand much efforts have been made to investigate the effects of inelastic e-p scattering on electronic transport through different nanostructures,$\frac{16-24}{2}$ on the other hand many theoretical works have devoted to understanding the phenomena of heat, various models and methods were suggested as well ${ }^{25-36}$ By using the nonequilibrium Green's function (NEGF) method, a tractable formula of heat generation has been derived $\underline{28}$ But these works mainly focused on the steady-state cases or, at most, the sinusoidal-like ac bias. In order to analyze the transient heat generation and its dynamics, the step-like and the square-shaped pulse biases are considered as the optimal driving forces $\stackrel{37-39}{-}$ It is not only because they can provide the unambiguous time scales and the transient behaviours of heat generation, but also because any information in computers is presented in bytes that consist of a long series of " 0 " and " 1 " digits, they are realized by continuously switching on and off the sharp step pulse.

In this paper, we study the transient behaviours of heat generation in a quantum dot (QD) system driven by a step-like or a square-shaped pulse bias. The QD is described by the Anderson model and the intra-dot e-p interaction is considered. By using the NEGF method, the transient heat generation is derived. We find a sudden bias inducing a periodically oscillating heat generation. Moreover, it can give rise to an energy transfer from the zero-temperature phonon subsystem to the electronic subsystem under certain conditions. However, after the bias goes back to zero and the system restores the equilibrium, the total heat generation at zero-temperature is always positive because of the third law of thermodynamics. In addition, we also discuss how to operate the QD with as little heat generation as possible.

The rest of the paper is organized as follows. In Sec. II we describe the model and deduce the formula of the transient heat generation. In Sec. [II] we give the numerical results and discussion. Finally, a brief conclusion is presented in Sec. IV

\section{THEORETICAL MODEL}

To begin with, we consider a single-level QD connecting to two electrode-leads and coupling to a local vibronic phonon mode. The whole system is described by the typical Hamiltonian:28

$$
\begin{aligned}
H & =\omega_{q} \hat{a}_{q}^{\dagger} \hat{a}_{q}+\lambda_{q}\left[\hat{a}_{q}+\hat{a}_{q}^{\dagger}\right] \hat{n}_{d}+\epsilon_{d} \hat{n}_{d} \\
& +\sum_{\alpha, k} \epsilon_{\alpha k}(t) \hat{c}_{\alpha k}^{\dagger} \hat{c}_{\alpha k}+\sum_{\alpha, k} t_{\alpha}\left[\hat{c}_{\alpha k}^{\dagger} \hat{d}+\text { H.c. }\right]
\end{aligned}
$$

Herein, $\hat{n}_{d}=\hat{d}^{\dagger} \hat{d}, \hat{d}$ and $\hat{c}_{\alpha k}(\alpha=\mathrm{L}, \mathrm{R})$ are electronic annihilation operators in the QD and lead $\alpha, \epsilon_{d}$ and $\epsilon_{\alpha k}(t)$ are the corresponding single-particle energies, and $t_{\alpha}$ represents the hopping matrix element between the QD and lead $\alpha$. As for the phonon subsystem, $\hat{a}_{q}\left(\hat{a}_{q}^{\dagger}\right)$ is an annihilation (creation) operator for one specific vibronic mode 
with frequency $\omega_{q}$. The strength of the e-p interaction is described by the coupling constant $\lambda_{q}$.

Due to the pulse bias, the electronic level $\epsilon_{\alpha k}(t)$ in Eq. (11) is time-dependent: $\epsilon_{\alpha k}(t)=\epsilon_{\alpha k}+\Delta_{\alpha}(t)$, and $\Delta_{\alpha}(t)$ represents the external time-dependent bias potential. For the step-like pulse case, we assume that the system is initially in equilibrium under the zero bias; at $t=0$ a bias $V$ is suddenly switched on and remains for $t>0$, which drives the system out of equilibrium, induces the electric current and then generates heat. Following we study the transient heat generation for $t>0$. Hereafter we consider that the bias is applied solely on the left lead, so $\Delta_{\mathrm{R}}(t)=0, \Delta_{\mathrm{L}}(t)=0$ for $t<0$, and $\Delta_{\mathrm{L}}(t)=V$ otherwise.

In what follows, it is rewarding to make a canonical transformation on the Hamiltonian $\bar{H}=U H U^{\dagger}$, with the unitary operator $U=\exp \left[\left(\lambda_{q} / \omega_{q}\right)\left(\hat{a}_{q}^{\dagger}-\hat{a}_{q}\right) \hat{d}^{\dagger} \hat{d}\right]$ :

$$
\begin{aligned}
\bar{H} & =\omega_{q} \hat{a}_{q}^{\dagger} \hat{a}_{q}+\tilde{\epsilon}_{d} \hat{n}_{d} \\
& +\sum_{\alpha, k} \epsilon_{\alpha k}(t) \hat{c}_{\alpha k}^{\dagger} \hat{c}_{\alpha k}+\sum_{\alpha, k} t_{\alpha}\left[\hat{c}_{\alpha k}^{\dagger} \hat{d} \hat{X}+\text { H.c. }\right]
\end{aligned}
$$

where $\tilde{\epsilon}_{d}=\epsilon_{d}-\lambda_{q}^{2} / \omega_{q}$ is the renormalized electronic level of the QD, and the operator $\hat{X}=\exp \left[-\left(\lambda_{q} / \omega_{q}\right)\left(\hat{a}_{q}^{\dagger}-\hat{a}_{q}\right)\right]$. We use the general formula of transient heat generation presented in Ref. 28, which remains unchange under the canonical transformation:

$$
\begin{aligned}
Q(t) & =-2 \operatorname{Im} \omega_{q} \lambda_{q}^{2} \int_{-\infty}^{t} d t_{1} e^{-\mathrm{i} \omega_{q}\left(t_{1}-t\right)}\{ \\
& -N_{q}\left\langle\left\langle\hat{n}_{d}(t) \mid \hat{n}_{d}\left(t_{1}\right)\right\rangle\right\rangle^{r}+\left\langle\left\langle\hat{n}_{d}(t) \mid \hat{n}_{d}\left(t_{1}\right)\right\rangle\right\rangle\langle\} .
\end{aligned}
$$

Next we replace the operator $\hat{X}$ by its expectation value $\langle\hat{X}\rangle=\exp \left[-\left(\lambda_{q} / \omega_{q}\right)^{2}\left(N_{q}+1 / 2\right)\right]$, which is reasonable under the conditions of both $t_{\alpha} \ll$ $\lambda_{q}$ and $t_{\alpha} \gg \lambda_{q} \stackrel{18,40,41}{ }$ After this approximation, the electron and the phonon subsystems are decoupled. So we can rigorously employ the Wick's theorem to deal with the two-particle Green's function: $\left\langle T_{c}\left[\hat{n}_{d}(t) \hat{n}_{d}\left(t_{1}\right)\right]\right\rangle_{\bar{H}}=\left\langle T_{c}\left[\hat{n}_{d}(t)\right]\right\rangle_{\bar{H}}\left\langle T_{c}\left[\hat{n}_{d}\left(t_{1}\right)\right]\right\rangle_{\bar{H}}-$ $\left\langle T_{c}\left[\hat{d}^{\dagger}(t) \hat{d}\left(t_{1}\right)\right]\right\rangle_{\bar{H}}\left\langle T_{c}\left[\hat{d}^{\dagger}\left(t_{1}\right) \hat{d}(t)\right]\right\rangle_{\bar{H}}$. Then the heat generation $Q(t)$ changes into:

$$
\begin{aligned}
Q(t) & =2 \operatorname{Re} \omega_{q} \lambda_{q}^{2} \int_{-\infty}^{t} d t_{1} e^{-\mathrm{i} \omega_{q}\left(t_{1}-t\right)}\{ \\
& -N_{q}\left[\widetilde{G}^{r}\left(t, t_{1}\right) \widetilde{G}^{<}\left(t_{1}, t\right)+\widetilde{G}^{<}\left(t, t_{1}\right) \widetilde{G}^{a}\left(t_{1}, t\right)\right] \\
& \left.+\left[\widetilde{G}^{<}\left(t, t_{1}\right) \widetilde{G}^{>}\left(t_{1}, t\right)-\widetilde{G}^{<}(t, t) \widetilde{G}^{<}\left(t_{1}, t_{1}\right)\right]\right\},(4)
\end{aligned}
$$

where $\widetilde{G}^{r, a,<,>}\left(t, t_{1}\right)$ are the standard single-particle Green's functions of the QD derived from the decoupled Hamiltonian. $\left.N_{q}=1 /\left[\exp \left(\omega_{q} / k_{\mathrm{B}} T\right)-1\right)\right]$ is the Bose distribution function with temperature $T$. Then by considering the zero temperature case $(T=0)$, the expression of transient heat generation reduces to:

$$
\begin{aligned}
Q(t) & =2 \operatorname{Re} \omega_{q} \lambda_{q}^{2} \int_{-\infty}^{t} d t_{1} e^{-\mathrm{i} \omega_{q}\left(t_{1}-t\right)} \\
& \times\left[\widetilde{G}^{<}\left(t, t_{1}\right) \widetilde{G}^{>}\left(t_{1}, t\right)-\widetilde{G}^{<}(t, t) \widetilde{G}^{<}\left(t_{1}, t_{1}\right)\right],
\end{aligned}
$$

and the Green's functions $\widetilde{G}^{<,>}$take the forms as: ${ }^{42,43}$

$$
\begin{aligned}
\widetilde{G}^{<}\left(t, t_{1}\right)= & \sum_{\alpha} \mathrm{i} \widetilde{\Gamma}_{\alpha} \int \frac{d \epsilon}{2 \pi} f(\epsilon) \widetilde{A}_{\alpha}(\epsilon, t) \widetilde{A}_{\alpha}^{*}\left(\epsilon, t_{1}\right) \\
& \times \exp \left[-\mathrm{i} \epsilon\left(t-t_{1}\right)-\mathrm{i} \int_{t_{1}}^{t} d t^{\prime} \Delta_{\alpha}\left(t^{\prime}\right)\right], \\
\widetilde{G}^{>}\left(t, t_{1}\right)= & \widetilde{G}^{<}\left(t, t_{1}\right)+\widetilde{G}^{r}\left(t, t_{1}\right)-\left[\widetilde{G}^{r}\left(t, t_{1}\right)\right]^{*}
\end{aligned}
$$

In Eq. (6), the linewidth function $\widetilde{\Gamma}_{\alpha}=\Gamma_{\alpha}\langle\hat{X}\rangle^{2}, \Gamma_{\alpha}=$ $2 \pi \rho_{\alpha}\left|t_{\alpha}\right|^{2}$, with the density of state $\rho_{\alpha}$ of lead $\alpha . f(\epsilon)=$ $1 /\left\{\exp \left[(\epsilon-\mu) / k_{\mathrm{B}} T\right]+1\right\}$ is the Fermi distribution function. To avoid trivial details, we have introduced the quantity $\widetilde{A}_{\alpha}(\epsilon, t)$ defined as: $\underline{42,43}$

$$
\begin{aligned}
\widetilde{A}_{\alpha}(\epsilon, t)= & \int_{-\infty}^{t} d t^{\prime} \widetilde{G}^{r}\left(t, t^{\prime}\right) \\
& \times \exp \left[\mathrm{i} \epsilon\left(t-t^{\prime}\right)+\mathrm{i} \int_{t^{\prime}}^{t} d t^{\prime \prime} \Delta_{\alpha}\left(t^{\prime \prime}\right)\right] .
\end{aligned}
$$

The retarded Green's function $\widetilde{G}^{r}\left(t, t^{\prime}\right)=-\mathrm{i} \theta(t-$ $\left.t^{\prime}\right) \exp \left[-\mathrm{i} \tilde{\epsilon}_{d}\left(t-t^{\prime}\right)-\widetilde{\Gamma}\left(t-t^{\prime}\right)\right]$ can be easily derived, in which $\widetilde{\Gamma}=\left(\widetilde{\Gamma}_{\mathrm{L}}+\widetilde{\Gamma}_{\mathrm{R}}\right) / 2$. It is independent of the bias potential $\Delta_{\alpha}(t)$. Combining the Eqs. (5 8), the transient heat generation $Q(t)$ can be calculated for arbitrary timedependent bias $\Delta_{\alpha}(t)$. Once $Q(t)$ is known, the total heat generation $Q_{T}(t)$ from $t=0$ to the time $t$ is straightforwardly obtained as: $Q_{T}(t)=\int_{-\infty}^{t} d t^{\prime} Q\left(t^{\prime}\right)=\int_{0}^{t} d t^{\prime} Q\left(t^{\prime}\right)$. Concretely, by taking into account of the step-like pulse bias, $\widetilde{A}_{\alpha}(\epsilon, t)$ is to be simplified:

$$
\begin{aligned}
\widetilde{A}_{\mathrm{R}}(\epsilon, t)= & \widetilde{G}^{r}(\epsilon)=1 /\left(\epsilon-\tilde{\epsilon}_{d}+\mathrm{i} \widetilde{\Gamma}\right) \\
\widetilde{A}_{\mathrm{L}}(\epsilon, t<0)= & \widetilde{G}^{r}(\epsilon) \\
\widetilde{A}_{\mathrm{L}}(\epsilon, t>0)= & \widetilde{G}^{r}(\epsilon+V)+\left[\widetilde{G}^{r}(\epsilon)-\widetilde{G}^{r}(\epsilon+V)\right] \\
& \times \exp \left[\mathrm{i}\left(\epsilon+V-\tilde{\epsilon}_{d}+\mathrm{i} \widetilde{\Gamma}\right) t\right] .
\end{aligned}
$$

In the following calculation, we will set the chemical potential $\mu=0$ of the equilibrium system at $t<0$ as the zero point of energy, and also assume the symmetric barriers $\widetilde{\Gamma}_{\mathrm{L}}=\widetilde{\Gamma}_{\mathrm{R}}$.

\section{RESULTS AND DISCUSSION}

Fig. 1(a) shows the transient heat generation $Q(t)$ versus the time $t$ with fixed $V=1$ and different renormalized level $\tilde{\epsilon}_{d}$. While $t<0$, the bias is zero and the system is in equilibrium, so one always has $Q(t)=0$. At $t=0$, the bias is suddenly added, then the current $I(t)$ and the heat generation $Q(t)$ emerge at $t>0$. $Q(t)$ displays a periodically oscillating behaviour with the period $2 \pi / \omega_{q}$. The oscillation is undamped and keeps all along due to only a discrete phonon model being considered here. $Q(t)$ is positive in one half period and negative in other half period, which means that the transporting electron periodically emits and absorbs phonon. Besides, the oscillating amplitude is sensitive to $\tilde{\epsilon}_{d}$, it is the largest while $\tilde{\epsilon}_{d}=0$ because of the occurrence of resonant tunnelling 

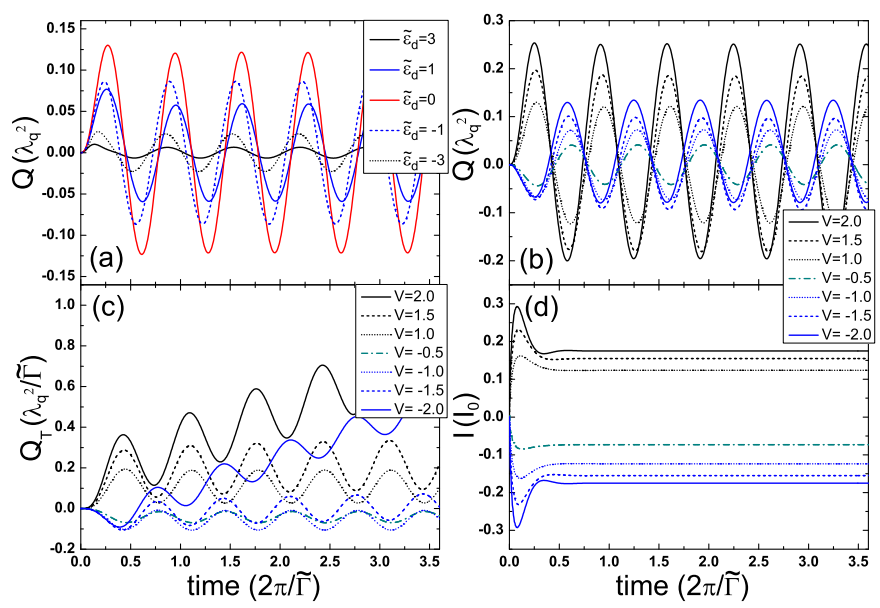

FIG. 1: (Colour online) The transient behaviours in response to a sudden step-like pulse bias with phonon subsystem having a single vibronic mode. (a) $Q(t)$ vs. $t$ with $V=1$. (b), (c) and (d) are respectively $Q(t), Q_{T}(t)$, and $I(t)$ vs. $t$ with $\tilde{\epsilon}_{d}=0$. Other parameters are $\omega_{q}=1.5$ and $\widetilde{\Gamma}=1$. The unit $I_{0}=e \widetilde{\Gamma} / \hbar$.

with the largest current for that $\tilde{\epsilon}_{d}$. When $\tilde{\epsilon}_{d}$ deviates from zero, the oscillating amplitude reduces and gradually approaches zero.

Next we fix the level $\tilde{\epsilon}_{d}=0$ and investigate the behaviours of $Q(t)$ with different bias $V$. For the positive bias $V, Q(t)$ is positive in the pre-half period and negative in the post-half period. But for the negative $V, Q(t)$ can be negative in the pre-half period [see the region of $0<t<\pi / \omega_{q} \approx 0.33(2 \pi / \widetilde{\Gamma})$ in Fig. [1(b)]. This result is surprising and indicates that the suddenly-added bias causes the phonon to be transferred from the phonon subsystem to the electronic subsystem at once. Notice that the temperature of the system has been set equal to zero, so it means the phonon population can be less than the original zero-T value. With $|V|$ increasing, the oscillating amplitude of $Q(t)$ also increases. For the positive- $Q$ half period, the amplitude increase is very prominent. But for the negative- $Q$ half period, the amplitude increase is small, and even decreases for the large $|V|$. So in the case of large $|V|\left(|V|>\omega_{q}\right)$, more phonons are emitted than absorbed, which leads to a net heat generation by the current.

In Fig. 1(c), the total heat generation $Q_{T}(t)$ is shown. Because of the oscillating $Q(t), Q_{T}(t)$ also exhibits the oscillating property with time $t$. For the small bias $\left(0<|V|<\omega_{q}\right), Q_{T}(t)$ oscillates along a horizontal line, in which case there is no net heat generation. But for the large bias $\left(|V|>\omega_{q}\right)$, an ascending trend will grow more and more noticeably, by considering the large time scale, $Q_{T}(t)$ is approximately proportional to $t$. In particular, as for $-\omega_{q}<V<0$, the curves of $Q_{T}(t)$ always lie below the zero point [see the curves with $V=-0.5$ and -1.0 in Fig. 1(c)], which means that at any time there is a net phonon absorption from the zero- $T$ phonon subsystem.

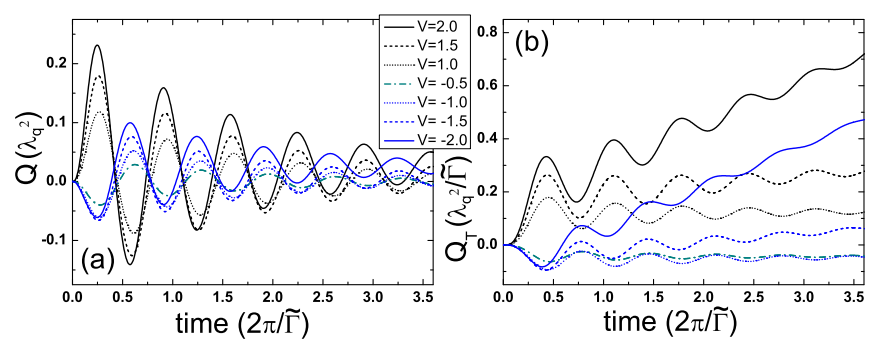

FIG. 2: (Colour online) The transient behaviours in response to a sudden step-like pulse bias with phonon subsystem having a Lorentzian spectrum. (a) and (b) are $Q(t)$ and $Q_{T}(t)$ respectively. The parameters are $\tilde{\epsilon}_{d}=0, \omega_{q}^{0}=1.5, \sigma=0.1$, and $\widetilde{\Gamma}=1$.

Intuitively, this seems to be impossible. But in fact, due to the e-p interaction, the equilibrium phonon population $n_{\mathrm{ph}}=\left\langle a_{q}^{\dagger}(t) a_{q}(t)\right\rangle$ at $t<0$ is not zero, but a finite value even if $T=0$. By using the NEGF method and taking the same approximation as in the derivation of $Q(t), n_{\mathrm{ph}}$ can be calculated. It is a function of $\tilde{\epsilon}_{d}$ behaving like the occupation number of electron on the QD, and we will mention it later in this paper (see Fig. 31). Then by choosing the same parameters in Fig. 1(c), $n_{\mathrm{ph}}$ is about $0.13 \lambda_{q}^{2} / \widetilde{\Gamma}^{2}$. From the above results, we find that the suddenly-applied bias can transfer a part of energy of such $n_{\mathrm{ph}}$ phonons from the original zero- $\mathrm{T}$ phonon subsystem to the electronic subsystem. Due to the system at $t>0$ being in non-equilibrium, this transfer is not prohibited in principle. Furthermore, we have already checked that the total heat absorption $-Q_{T}(t)$ from the phonon subsystem does not exceed the value of $\omega_{q} n_{\mathrm{ph}} \approx 0.20 \lambda_{q}^{2} / \widetilde{\Gamma}$ in any case.

The response of transient electric current $I(t)$ to the sudden pulse bias is also shown in Fig. 1(d) $\stackrel{44}{\longleftarrow}$ in which $I(t)$ quickly rises in a very short time. The rising time scale of $I(t)$ is about $0.1(2 \pi / \widetilde{\Gamma})$. However, the rise of $Q(t)$ is within the time scale $(1 / 4)\left(2 \pi / \omega_{q}\right)$. So if we set the parameter $\widetilde{\Gamma}>0.4 \omega_{q}$, the current $I(t)$ will emerge much quicker than $Q(t)$. In this case, the device can finish the operation before the occurrence of heat generation. Additionally, in comparison with $Q(t), 1) I(t)$ is without oscillation and quickly damps to a stable value because of the spectrum of electronic reservoirs (lead L and R) being continuous, 2) $I(t)$ has the electron-hole symmetry [e.g. $\left.I\left(t,-V,-\tilde{\epsilon}_{d}\right)=-I\left(t, V, \tilde{\epsilon}_{d}\right)\right]$, but $Q\left(t,-V,-\tilde{\epsilon}_{d}\right) \neq$ $\pm Q\left(t, V, \tilde{\epsilon}_{d}\right)$, it is because that the phonon subsystem is without the electron-hole symmetry.

Up to now, we have only considered a single isolated phonon mode $\omega_{q}$ in the QD. But for a real device, the QD usually couples to the lead and the substrate, so the phonon mode $\omega_{q}$ will unavoidably acquire a small line width. This is to be considered in the following discussion with the phonon subsystem having a Lorentzian 


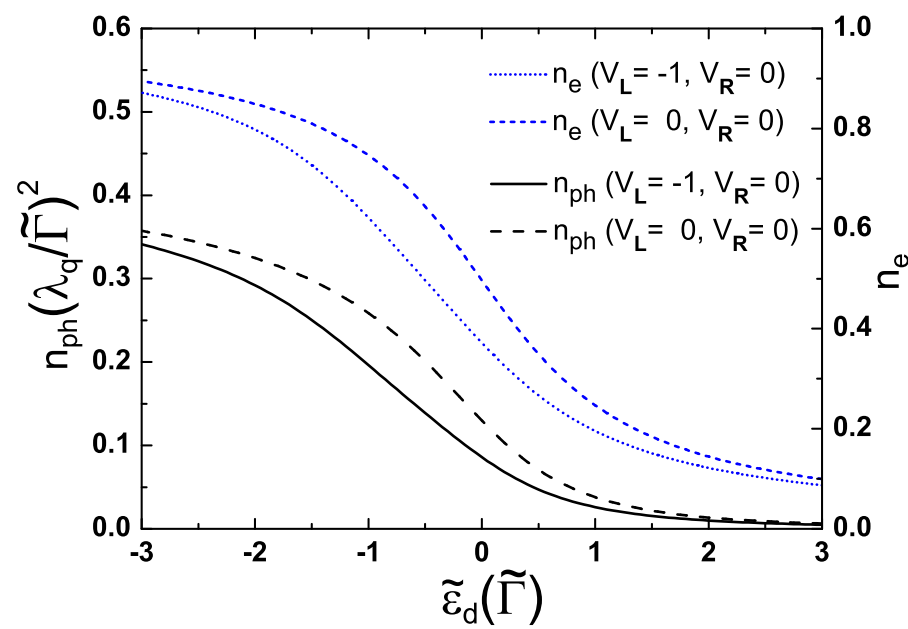

FIG. 3: (Colour online) The occupation number $n_{\mathrm{ph}}$ of phonon and the occupation number $n_{\mathrm{e}}$ of electron on the QD at zero temperature with respect to the renormalized electronic level $\tilde{\epsilon}_{d}$. Both the equilibrium-state case $\left(V_{\mathrm{L}}=0, V_{\mathrm{R}}=\right.$ $0)$ and the steady-state case $\left(V_{\mathrm{L}}=-1, V_{\mathrm{R}}=0\right)$ are considered. The parameters are set as $\omega_{q}=1.5, \widetilde{\Gamma}=1$.

spectrum:

$$
A\left(\omega_{q}\right)=\frac{\theta\left(\omega_{q}\right)}{\pi} \frac{\sigma}{\left(\omega_{q}-\omega_{q}^{0}\right)^{2}+\sigma^{2}}
$$

Now the oscillations of $Q(t)$ and $Q_{T}(t)$ gradually damp as shown in Fig. 2, $Q(t)$ damps to a finite positive value for $|V| \geq \omega_{q}^{0}$ or almost zero for $|V|<\omega_{q}^{0}$. $Q_{T}(t)$ damps along a slanting line for $|V| \geq \omega_{q}^{0}$ or a horizontal line for $|V|<$ $\omega_{q}^{0}$. In particular, for $-\omega_{q}^{0}<V<0, Q(t) \approx 0$ and $Q_{T}(t)$ is negative after the system reaches a new steady state at the large time $t$ [see the curves with $V=-0.5$ and $V=-1.0$ in Fig. 2(b)]. It means that, in the process of applying the sudden bias, a net energy transfer from the phonon subsystem to the electronic subsystem occurs. The phonon population $n_{\mathrm{ph}}$ in the new steady state is less than that of the original zero- $T$ case and it can keep this value for a very long time because of $Q(t) \approx 0$. To pay attention, the system is now in the steady state, not in equilibrium. So $n_{\mathrm{ph}}$ being less than the original zero$\mathrm{T}$ value does not indicate that its temperature is lower than 0 , and this result does not breach the third law of thermodynamics either.

In order to further explain and comprehend the phenomenon of heat absorption from the phonon subsystem at zero temperature, we have calculated the occupation number of zero- $T$ phonon, $n_{\mathrm{ph}}$, and the occupation number of electron, $n_{\mathrm{e}}$, in both equilibrium state and steady

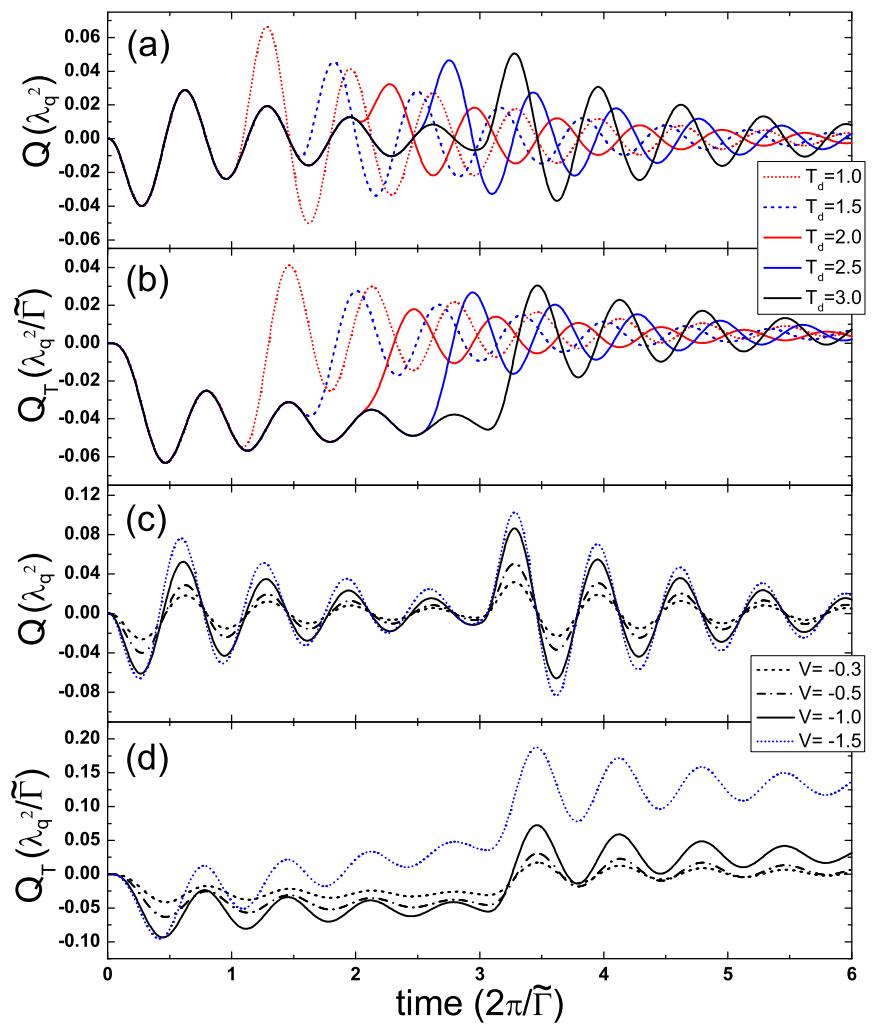

FIG. 4: (Colour online) The transient behaviours in response to a square-shaped pulse bias with phonon subsystem having a Lorentzian spectrum. (a) and (c) show $Q(t)$ vs. $t$, (b) and (d) are $Q_{T}(t)$ vs. $t$. In (a) and (b), $V=-0.5$, in (c) and (d), $T_{d}=3$. Other parameters are the same as those in Fig. 2.

state:

$$
\begin{aligned}
n_{\mathrm{e}} & =\left\langle\hat{n}_{d}\right\rangle=-\mathrm{i} \int \widetilde{G}^{<}(\epsilon) d \epsilon / 2 \pi \\
n_{\mathrm{ph}} & =\frac{\lambda_{q}^{2}}{4 \pi^{2}} \iint d \epsilon_{1} d \epsilon_{2} \\
& \times\left[\frac{\widetilde{G}^{<}\left(\epsilon_{1}\right) \widetilde{G}^{>}\left(\epsilon_{2}\right)}{\left(\epsilon_{1}-\epsilon_{2}-\omega_{q}\right)^{2}}-\frac{\widetilde{G}^{<}\left(\epsilon_{1}\right) \widetilde{G}^{<}\left(\epsilon_{2}\right)}{\omega_{q}^{2}}\right],
\end{aligned}
$$

where $\quad \widetilde{G}^{<}(\epsilon)=\mathrm{i} \widetilde{G}^{r}(\epsilon)\left[\sum_{\alpha} \widetilde{\Gamma}_{\alpha} f_{\alpha}(\epsilon)\right] \widetilde{G}^{a}(\epsilon)$, $\widetilde{G}^{>}(\epsilon)=-\mathrm{i} \widetilde{G}^{r}(\epsilon)\left\{\sum_{\alpha} \widetilde{\Gamma}_{\alpha}\left[1-f_{\alpha}(\epsilon)\right]\right\} \widetilde{G}^{a}(\epsilon), \quad$ and $f_{\alpha}(\epsilon)=1 /\left\{\exp \left[\left(\epsilon-\mu_{\alpha}\right) / k_{\mathrm{B}} T\right]+1\right\} \quad(\alpha=\mathrm{L}, \mathrm{R})$. See Fig. 3, the occupation number of phonon behaves much like that of electron, which monotonically decreases with the elevated renormalized level of the QD, that is to say, $n_{\mathrm{ph}}$ is approximately proportional to $n_{\mathrm{e}}$ while changing $\tilde{\epsilon}_{d}$. At $t<0$, there is no bias across the QD $\left(V_{\mathrm{L}}=0, V_{\mathrm{R}}=0\right)$, so the whole system is in equilibrium. After applying the sudden bias, the system is driven out of equilibrium, until a large time, enters a new steady state $\left(V_{\mathrm{L}}=-1, V_{\mathrm{R}}=0\right)$. As we can see, both the phonon population $n_{\mathrm{ph}}$ and the electron population $n_{\mathrm{e}}$ experience an obvious falling down from the equilibrium-state case to the steady-state case, it means that a part of zero- $T$ phonons have indeed run away, 
which results in the heat absorption. To be specific, for $\tilde{\epsilon}_{d}=0$, the change of the average phonon number at zero temperature is about $0.044 \lambda_{q}^{2} / \widetilde{\Gamma}^{2}$, and the corresponding phonon energy is $0.066 \lambda_{q}^{2} / \widetilde{\Gamma}$. As a matter of fact, the net heat absorption $-Q_{T}$ in real case will be a little less than this value, in that the electric current can also transfer energy to the phonon subsystem as an offset [see the approached level of $Q_{T}$ with $V=-1.0$ in Fig. 2(b)].

Finally, we deal with the square-shaped pulse bias $\Delta_{\mathrm{L}}(t)$, in which case $\Delta_{\mathrm{L}}(t)$ suddenly goes back to 0 at $t=T_{d}$. Fig. 团 shows $Q(t)$ and $Q_{T}(t)$ for the Lorentzianspectrum phonon subsystem with different $T_{d}$ and $V$. While the bias going back to $0, Q(t)$ and $Q_{T}(t)$ show strong oscillations once again, which will gradually fade away. In the limit of $(t \rightarrow \infty)$, the system restores the equilibrium as initial, $Q(t)$ and $Q_{T}(t)$ approaches zero and a finite positive value respectively. For $|V| \geq \omega_{q}^{0}$, $Q_{T}(\infty)$ is a large value that is approximately proportional to $T_{d}$. But for $|V|<\omega_{q}^{0} / 2, Q_{T}(\infty)$ is almost zero regardless of $T_{d}$. So if the device is operated in that range of $|V|$, there will not be much net heat generation. In particular, as discussed above, for $-\omega_{q}^{0}<V<0, Q_{T}(t)$ can be negative after the first pulse bias, but $Q_{T}$ is eventually $\geq 0$ when the system recovers the equilibrium state. Actually, because of the third law of thermodynamics, it is prohibited to absorb heat energy from the zero- $\mathrm{T}$ phonon subsystem under the condition of equilibrium, no matter how to control the bias.

\section{CONCLUSIONS}

In summery, the transient heat generation in the QD driven by a step-like pulse bias is investigated. We find that the suddenly switched-on bias can induce a heat absorption from the zero-temperature phonon subsystem. For a strong QD-lead coupling case, the current-rising process is much quicker than the heat generation, so the device can finish the electronic operation before the emergence of heat generation. In addition, for a small bias, the net heat generation is always very small no matter how to turn on and off the device.

\section{ACKNOWLEDGEMENTS}

This work was financially supported by NSF-China under Grants Nos. 10821403, 10974236, and 11074174, China-973 program and US-DOE under Grants No. DEFG02- 04ER46124.
* Electronic address: sunqf@iphy.ac.cn

1 R. H. M. Smit, C. Untiedt, and J. M. van Ruitenbeek, Nanotechnology 15, S472 (2004).

2 M. Tsutsui, S. Kurokawa, and A. Sakai, Nanotechnology 17, 5334 (2006).

3 Z. F. Huang, B. Q. Xu, Y. C. Chen, M. Di Ventra, and N. J. Tao, Nano Lett. 6, 1240 (2006).

4 Z. F. Huang, F. Chen, R. D'Agosta, P. A. Bennett, M. Di Ventra, and N. J. Tao, Nature Nano. 2, 698 (2007).

5 M. Tsutsui, M. Taniguchi, and T. Kawai, Nano Lett. 8, 3293 (2008).

${ }^{6}$ M. Tsutsui, M. Taniguchi, K. Yokota, and T. Kawai, Appl. Phys. Lett. 96, 103110 (2010).

7 M. Oron-Carl and R. Krupke, Phys. Rev. Lett. 100, 127401 (2008).

8 Z. Ioffe, T. Shamai, A. Ophir, G. Noy, I. Yutsis, K. Kfir, O. Cheshnovsky, and Y. Selzer, Nature Nano. 3, 727 (2008).

${ }^{9}$ H. Park, J. Park, A. K. L. Lim, E. H. Anderson, A. P. Alivisatos, and P. L. McEuen, Nature 407, 57 (2000).

10 B. J. LeRoy, S. G. Lemay, J. Kong, and C. Dekker, Nature 432, 371 (2004).

11 B. J. LeRoy, J. Kong, V. K. Pahilwani, C. Dekker, and S. G. Lemay, Phys. Rev. B 72, 075413 (2005).

12 S. Sapmaz, P. Jarillo-Herrero, Y. M. Blanter, C. Dekker, and H. S. J. van der Zant, Phys. Rev. Lett. 96, 026801 (2006).

13 A. K. Hüttel, B. Witkamp, M. Leijnse, M. R. Wegewijs, and H. S. J. van der Zant, Phys. Rev. Lett. 102, 225501 (2009).

14 R. Leturcq, C. Stampfer, K. Inderbitzin, L. Durrer, C. Hierold, E. Mariani, M. G. Schultz, F. von Oppen, and K. Ensslin, Nature Phys. 5, 327 (2009).

15 G. A. Steele, A. K. Hüttel, B. Witkamp, M. Poot, H. B. Meerwaldt, L. P. Kouwenhoven, and H. S. J. van der Zant,
Science 325, 1103 (2009).

16 M. J. Montgomery, J. Hoekstra, T. N. Todorov, and A. P. Sutton, J. Phys.: Condens. Matter 15, 731 (2003).

17 Y.-C. Chen, M. Zwolak, and M. Di Ventra, Nano Lett. 4, 1709 (2004).

18 Z.-Z. Chen, R. Lü, and B.-F. Zhu, Phys. Rev. B 71, 165324 (2005).

19 M. Paulsson, T. Frederiksen, and M. Brandbyge, Phys. Rev. B 72, 201101(R) (2005).

20 M. Galperin, M. A. Ratner, and A. Nitzan, J. Phys.: Condens. Matter 19, 103201 (2007).

21 W. Rudziński, J. Phys.: Condens. Matter 20, 275214 (2008)

22 R.-P. Riwar and T. L. Schmidt, Phys. Rev. B 80, 125109 (2009).

23 M. Tahir and A. MacKinnon, Phys. Rev. B 81, 195444 (2010).

24 T.-F. Fang, Q.-F. Sun, and H.-G. Luo, Phys. Rev. B 84, 155417 (2011).

25 Y.-C. Chen, M. Zwolak, and M. Di Ventra, Nano Lett. 3, 1691 (2003).

26 M. Lazzeri, S. Piscanec, F. Mauri, A. C. Ferrari, and J. Robertson, Phys. Rev. Lett. 95, 236802 (2005).

27 A. P. Horsfield, D. R. Bowler, H. Ness, C. G. Sánchez, T. N. Todorov, and A. J. Fisher, Rep. Prog. Phys. 69, 1195 (2006).

28 Q.-F. Sun and X. C. Xie, Phys. Rev. B 75, 155306 (2007).

29 M. Galperin, A. Nitzan, and M. A. Ratner, Phys. Rev. B 75, 155312 (2007).

30 J. T. Lü and J.-S. Wang, Phys. Rev. B 76, 165418 (2007).

31 J. Liu, J. T. Song, Q.-F. Sun, and X. C. Xie, Phys. Rev. B 79, 161309(R) (2009).

32 B. H. Wu and J. C. Cao, J. Phys.: Condens. Matter 21, 245301 (2009). 
33 L.-L. Zhou, S. S. Li, J. N. Wei, and S. Q. Wang, Phys. Rev. B 83, 195303 (2011).

34 Z.-X. Xie, K.-Q. Chen, and W. H. Duan, J. Phys.: Condens. Matter 23, 315302 (2011).

35 V. L. Gurevich, V. I. Kozub, and M. I. Muradov, J. Phys.: Condens. Matter 23, 405302 (2011).

${ }^{36}$ F. Chi and Y. Dubi, J. Phys.: Condens. Matter 24, 145301 (2012).

37 M. Plihal, D. C. Langreth, and P. Nordlander, Phys. Rev. B 61, 13341(R) (2000).

38 J. Maciejko, J. Wang, and H. Guo, Phys. Rev. B 74, 085324 (2006).

${ }^{39}$ Y. X. Xing, Q.-F. Sun, and J. Wang, Phys. Rev. B 75,
$125308(2007)$

40 G. D. Mahan, Many-Particle Physics (Plenum, New York, 1990).

41 A. C. Hewson and D. M. Newns, J. Phys. C: Solid St. Phys. 13, 4477 (1980).

${ }^{42}$ H. Haug and A.-P. Jauho, Quantum Kinetics in Transport and Optics of Semiconductors (Springer-Verlag, Berlin, 1998).

43 A.-P. Jauho, N. S. Wingreen, and Y. Meir, Phys. Rev. B 50, 5528 (1994).

44 In the current calculation, we use the same method in Ref. 18 . 